\title{
Yogurt simbiótico con harina de maracuyá (Passiflora edulis) como apoyo nutricional en personas con hiperglicemia
}

\author{
Symbiotic yogurt and flour of passion fruit (Passiflora \\ edulis) as nutritional support in people with hyperglycemia
}

ID Oscar Otilio Osso Arriz, (iD Dennis Lazo Ramos $₫$

Universidad Le Cordon Bleu. Lima, Perú

\begin{tabular}{llll}
\hline Recibido: 29/04/2018 & Revisado: 12/05/2019 & Aceptado: 25/05/2019 & Publicado: 30/06/2019 \\
\hline
\end{tabular}

\section{RESUMEN}

El presente trabajo consiste en la elaboración de un yogurt simbiótico con harina de maracuyá (Passiflora edulis) como apoyo nutricional en personas con hiperglicemia tipo 2. A nivel metodológico, los métodos fueron un diseño experimental de corte lon-gitudinal, ya que se desarrolló un proceso de evaluación frente a un fenómeno que se estaba realizando, no observando.

El monitoreo de la hiperglicemia se midió al inicio y mensualmente en dos grupos que se comparan: grupo de casos que se le administró una cantidad diaria de yogurt simbiótico con harina de maracuyá y dieta, mientras que al otro grupo (control) recibió solo dieta, ambos durante tres meses.

Los resultados fueron que el tratamiento con dosis de $500 \mathrm{ml}$ por día produjo efectos positivos en la reducción entre el 8 y $9 \%$ de los valores cuantitativos de la hiperglicemia, con varia-ción significativa $(p<0,05)$ durante los tres meses de tratamiento, con mayor efectivi-dad que en el grupo control ( $p=0,110>0,05)$. El producto tiene buena aceptación com-parado con el yogurt natural $(p=0,194>0,05)$.

Se puede afirmar que existe interacción de la dieta y la reducción de la hiperglicemia tipo $2(0,000<0,05)$, sin embargo, se evi-dencia estadísticamente que es mayor cuando se asocia con el consumo de yogurt simbiótico con harina de maracuyá. Contribuye a una alimentación saludable com-plementaria a la dieta como apoyo nutricional en la hiperglicemia tipo 2.

Palabras clave: Yogur simbiótico, maracuyá harina, hiperglicemia tipo 2, diabetes. 


\section{ABSTRACT}

The objective is that symbiotic yogurt can be elaborate with passion fruit (Passiflora edulis) flour as nutritional support in people with type 2 hyperglycemia. The methods were experimental design, longitudinal cut, because an evaluation process was deve-loped against a phenomenon that was being carried out, not observing.

The monitoring of hyperglycemia was measured at the beginning and monthly in two groups that were compared: Group of cases that were given a daily amount of symbio-tic yogurt with passion fruit flour and diet, while the other group (control) received only diet, both for 3 months.

The results were that the treatment with a dose of $500 \mathrm{ml}$ per day produced positive effects in the reduction between $8 \%$ and $9 \%$ of the quanti-tative values of hyperglycemia, with significant variation $(p<0.05)$ during the 3 months of treatment, with greater effectiveness than in the control group $(p=0.110>0.05)$.

The product has good acceptance compared to natural yogurt ( $p=0.194>0.05)$. The conclusions are that there is interaction of diet and the reduction of type 2 hypergly-cemia $(0.000<0.05)$, however, it is statistically shown that it is higher when it is asso-ciated with the consumption of symbiotic yogurt with passion fruit flour. Contributes to a healthy diet complementary to the diet as nutritional support in type 2 hyperglyce-mia.

Keywords: Symbiotic yogurt, flour passion fruit, type 2 hyperglycemia, diabetes.

\section{INTRODUCCIÓN}

La diabetes tipo 2 es una enfermedad conocida como diabetes mellitus, que representa entre el 90 y $95 \%$ de todos los casos diagnosticados de diabetes.

En el Perú, un millón y medio de personas tienen diabetes tipo 2 y cada año se registran 100 mil nuevos casos, según el Ministerio de Salud (MINSA).

A nivel mundial cada seis segundos muere una persona a causa de la diabetes, cada tres se diagnóstica un nuevo caso y cada veinte alguien sufre una amputación por complicaciones relacionadas a esta enfermedad (OMS, 2017).

En ese sentido, el término "probiótico", es utilizado para designar a las bacterias que tienen efectos benéficos para los seres humanos y los animales (Metchnikoff, 1907), mientras que los "prebióticos" son componentes no digeribles de un alimento que al ingerirse promueven el crecimiento y establecimiento de gérmenes beneficiosos de la flora intestinal (Diplock A.T., 1999). Asimismo, se define como simbiótico a la mezcla de probióticos y prebióticos que generan un efecto en el huésped (Andersson H., 2001).

Contextualizando lo planteado y tomando en cuenta nuestra realidad, en el Perú, la cáscara de maracuyá se deshecha, donde se pierde abundante aporte de fibra dietética, que puede ayudar a mejorar la salud de las personas, por ello, en la presente investigación, se elaboró yogurt simbiótico con adición de harina de Passiflora edulis 
(maracuyá) como apoyo nutricional en personas con hiperglicemia tipo 2 .

Esto es con el fin de aprovechar los efectos del consumo de yogurt simbiótico y harina de maracuyá (Passiflora edulis) como apoyo nutricional en personas con hiperglicemia tipo 2 , orientada a la prevención de enfermedades degenerativas asociadas a la diabetes II.

Ante esto, se plantea el siguiente problema: ¿Será un apoyo nutricional significativo el yogurt simbiótico y harina de Passiflora edulis en personas con hiperglicemia tipo 2? y cuya hipótesis general es "a mayor ingesta de yogurt simbiótico con harina de maracuyá, en personas que reciben dieta controlada (del grupo de casos), será mayor la reducción de los niveles de hiperglicemia tipo 2, que solamente reciben dieta controlada (grupo de controles)".

\section{MATERIALES Y MÉTODOS}

Es un estudio experimental, en dos grupos de personas hiperglicemia tipo 2 que se comparan en un estudio de casos y controles, teniendo en cuenta los criterios de inclusión y exclusión. La selección fue aleatoria.

El trabajo corresponde a un diseño experimental, de corte longitudinal, porque las variables se midieron en cuatro momentos, con el fin de determinar la variación de los niveles de glicemia del grupo de casos (expuestos) y grupo de controles (no expuestos) durante cuatro meses.

Población: La presente investigación se realizó en personas con hiperglicemia tipo 2 , de 50 a 65 años, en la ciudad de Lima distrito de Miraflores.

Muestra:

Selección de los grupos: Adultos expuestos y no expuestos mayores de 50 años que presentaron hiperglicemia tipo 2, seleccionados según los criterios de inclusión. Grupo experimental: Quince (15) adultos mayores de 50 años hiperglicemia tipo 2 que consumieron yogurt simbiótico y harina de maracuyá con la dieta acostumbrada (del grupo de casos). Ellos fueron divididos en un grupo para la aplicación de la formulación elaborada.

Grupo control: Cinco (05) adultos mayores de 50 años hiperglicemia tipo 2 que solamente recibieron su dieta acostumbrada.

\section{Técnicas de recolección de datos}

a) Método de entrevista-interrogatorio: Aplicación de las encuestas.

b) Método de observación directa de los análisis bioquímicos de glucosa en sangre.

c) Fichaje durante el estudio y recopilación bibliográfica, según normas de la OMS.

\section{Técnicas para el procesamiento de la información}

Procedimiento de recolección: La autorización o permiso fue de la facultad de ciencias de los alimentos de la universidad Le Cordon Bleu. La autorización consentida se dio a los sujetos en estudio. El tiempo de recojo de la información, según el cronograma previsto, fue entre junio y octubre 2017.

Procesos: Seguidos durante el estudio. Entre octubre y diciembre del 2017, se invitaron a las personas a participar en el estudio.

Coordinación interna fue con el personal de apoyo para asegurar el cumplimiento del plan de recolección y para garantizar la validez y confiabilidad del estudio.

\section{RESULTADOS}

En primera instancia, podemos observar los resultados expuestos en la tabla 1: 
Tabla 1. Análisis físico químico de la harina de maracuyá

\begin{tabular}{cc}
\hline Componentes & Resultados \\
\hline Humedad g\% & 5,80 \\
Cenizas g\% & 6,50 \\
Grasa g\% & 0,75 \\
Proteínas g\% & 6,60 \\
pH & 5,40 \\
Acidez & 0,97 \\
Fibra soluble g\% & 3,50 \\
Fibra insoluble g\% & 54,19 \\
Glucosa & 3,01 \\
Sacarosa g\% & 7,54 \\
Hidratos de carbono g\% & 22,66 \\
Fenoles T. (mgEAG/gs) & $18,358 \pm 0,384$ \\
Antocianos T. (mgEC3G/gs) & $0,091 \pm 0.012$ \\
\hline mg equivalentes de ácido gálico/gramo de material seco \\
mg equivalentes a cianidina-3-glucósido por gramo de material seco
\end{tabular}

Por otro lado, en lo relacionado al análisis microbiológico, podemos indicar los si-guientes resultados:

Tabla 2. Análisis microbiológico de la harina de maracuyá

\begin{tabular}{|l|l|l|l|l|}
\hline \multicolumn{1}{|c|}{ Referencia } & \multicolumn{1}{|c|}{$\mathbf{1}$ día } & 30 días & \multicolumn{1}{c|}{ 60 días } & \multicolumn{1}{c|}{90 días } \\
\hline $\begin{array}{l}\text { Numeración de aeróbios } \\
\text { mesófilos viables } \\
\text { (UFC/g }=\mathrm{V}^{\circ} \mathrm{N}^{\circ} 10^{4}-10^{5^{*}}\end{array}$ & $<10$ & $<10$ & $<10$ & $<10$ \\
\hline $\begin{array}{l}\text { Numeración de salmonellas } \\
\text { (UFC/g) }=\mathrm{V}^{\circ} \mathrm{N}^{\circ}=<10^{3^{*}}\end{array}$ & 0 & 0 & 0 & 0 \\
\hline $\begin{array}{l}\text { Numeración de coliformes } \\
\text { (NMP/g) }=\mathrm{V}^{\circ} \mathrm{N}^{\circ}=<3^{*}\end{array}$ & 0 & 0 & 0 & 0 \\
\hline $\begin{array}{l}\text { Numeración de hongos } \\
\text { (UFC/g) }=\mathrm{V}^{\circ} \mathrm{N}^{\circ}=<10^{3^{*}}\end{array}$ & 0 & $<10$ & $<10$ & $<10$ \\
\hline
\end{tabular}

UFC= Unidad formadora de colonia NMP = Número más probables 
En la tabla 3, podemos ver el tratamiento con yogurt simbiótico y harina de maracuyá y dieta acostumbrada:

Tabla 3. Reducción de la hiperglicemia tipo 2 de la muestra, en el grupo de casos

\begin{tabular}{ccccccccc}
\hline Hiperglicemia & \multicolumn{2}{c}{ Inicio } & \multicolumn{2}{c}{ Primer mes } & \multicolumn{2}{c}{ Segundo mes } & \multicolumn{2}{c}{ Tercer mes } \\
& Cantidad & $\%$ & Cantidad & $\%$ & Cantidad & $\%$ & Cantidad & $\%$ \\
\hline Reducción & 15 & 100 & 5 & 33,3 & 11 & 61,1 & 14 & 93,3 \\
\hline
\end{tabular}

Por otro lado, podemos observar los resultados sin tratamiento con yogurt simbiótico y harina de maracuyá, solo dieta acostumbrada.

Tabla 4. Reducción de la hiperglicemia tipo 2 de la muestra, en el grupo control**

\begin{tabular}{ccccccccc}
\hline Hiperglicemia & \multicolumn{2}{c}{ Inicio } & \multicolumn{2}{c}{ Primer mes } & \multicolumn{2}{c}{ Segundo mes } & \multicolumn{2}{c}{ Tercer mes } \\
& Cantidad & $\%$ & Cantidad & $\%$ & Cantidad & $\%$ & Cantidad & $\%$ \\
\hline Reducción & 15 & 100 & 2 & 40,0 & 2 & 40,0 & 3 & 60,0 \\
\hline
\end{tabular}

Tabla 5. Estadísticos descriptivos de los niveles de glicemia en el grupo de casos

\begin{tabular}{crccc}
\hline \multirow{2}{*}{ Tiempo } & Media & Error típ. & \multicolumn{2}{c}{ Intervalo de confianza al 95\% } \\
\cline { 4 - 5 } & & & Límite inferior & Límite superior \\
\hline Inicio & 203,73 & 17,456 & 166,294 & 241,173 \\
& 3 & & & \\
Primer Mes & 146,66 & & & 170,980 \\
& 7 & 11,336 & 122,354 & 130,132 \\
Segundo Mes & 110,66 & & & 112,451 \\
\hline & 7 & 9,076 & 91,201 & \\
Tercer Mes & 95,600 & 7,857 & 78,749 & \\
\hline N = 15 & & & &
\end{tabular}

Se puede observar que la medida promedio de la glicemia de la muestra en el grupo de casos disminuyó en función directamente proporcional al tiempo de tratamiento con dosis de $500 \mathrm{ml}$ por día de yogurt simbiótico y harina de maracuyá, como apoyo nutricional a la dieta acostumbrada de personas con hiperglicemia tipo 2. Desde el primer mes se presentó efecto positivo siendo asimétrica hacía el límite superior con una dispersión de la reducción entre el $8 \%$ y $9 \%$ de la medida de los valores cuantitativos de la hiperglicemia. 
Tabla 6. Prueba de contraste multivariado.

\begin{tabular}{clc}
\hline Efecto & & Significación \\
\hline Tiempo & Traza de Pillai & 0,000 \\
& Lambda de Wilks & 0,000 \\
& Traza de Hotelling & 0,000 \\
& Raíz mayor de Roy & 0,000 \\
\hline
\end{tabular}

Según la tabla de contraste multivariado, el comportamiento de los niveles de la glicemia por efecto de la ingesta de $500 \mathrm{ml} /$ día de yogurt simbiótico y harina de maracuyá con respecto al tiempo de tratamiento en el grupo de casos, muestran que existen diferencias significativas $(p<0,05)$ en los cuatro periodos donde se tomaron las medidas de la glicemia en personas con hiperglicemia tipo 2, evidenciándose una asociación entre estas dos variables.

Tabla 7. Modelos de optimización de medida de glicemia

\begin{tabular}{clc}
\hline Fuente & \multicolumn{1}{c}{ tiempo } & Significación \\
\hline \multirow{2}{*}{ tiempo } & Lineal & 0,000 \\
& Cuadrático & 0,000 \\
& Cúbico & 0,992 \\
\hline
\end{tabular}

El análisis de regresión con la ecuación cuadrática $(p<0,05)$ obtenida a partir de los datos demuestra que existe una relación estadísticamente significativa entrelas variables (tratamiento y efecto sobre la hiperglicemia tipo 2) y proporciona un mejor ajuste que lo obtenido con la ecuación cúbica $(p>0,05)$. Por tal motivo, esta expresión es recomendable para hacer estimaciones y predicciones de los valores de la variable dependiente (porcentaje de personas que redujeron los niveles de hiperglicemia tipo 2) a partir de valores de la variable independiente (tiempo de tratamiento que consumieron la dosis de $500 \mathrm{ml}$ de yogurt simbiótico y harina de maracuyá, siendo más eficaz para optimizar o realizar una proyección del nivel de glicemia en personas con hiperglicemia tipo 2 . 


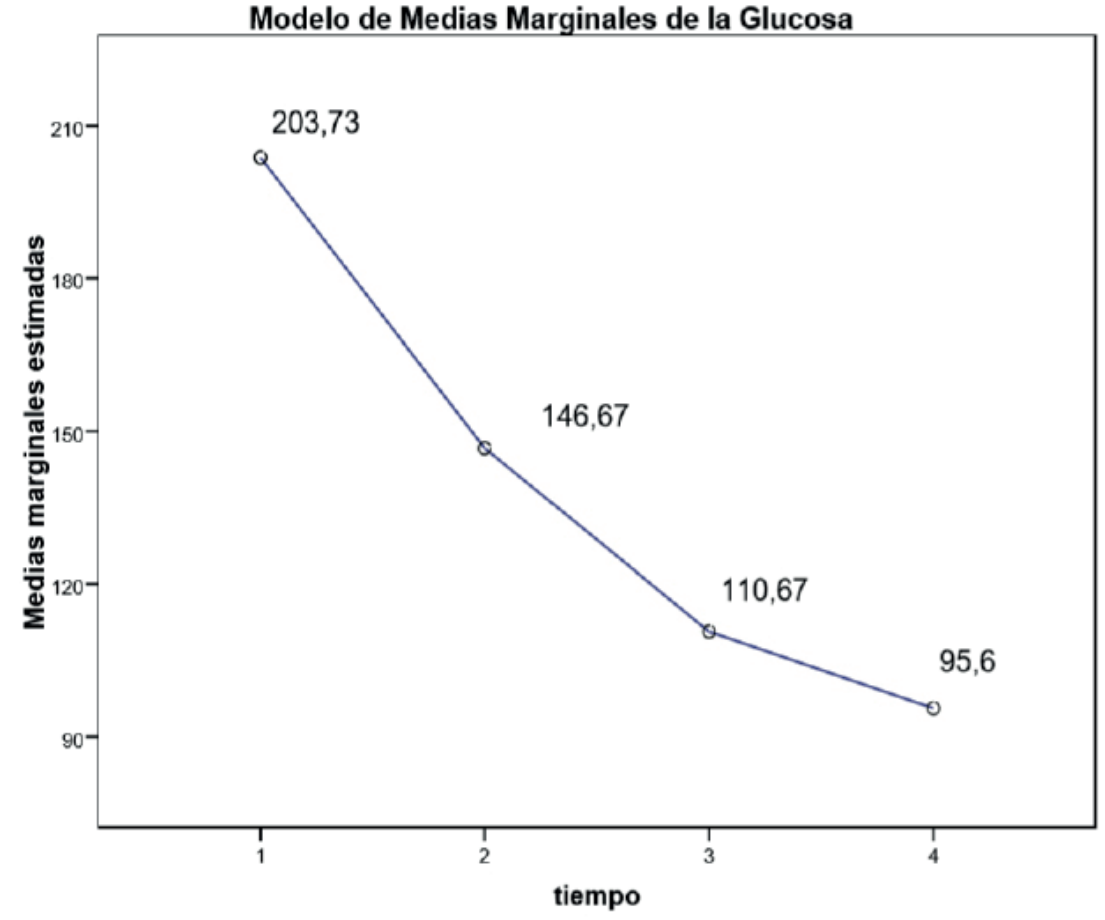

Figura 1. Comportamiento de los niveles de glicemia.

En la figura 1, se muestra que el comportamiento del modelo de regresión tiene tendencia cuadrática negativa. A su vez, evidencia cómo los promedios de los niveles de la glicemia en personas con hiperglicemia tipo 2 ha disminuido con la ingesta de $500 \mathrm{ml} /$ día de yogurt simbiótico y harina de maracuyá con respecto al tiempo de tratamiento.

Tabla 8. Prueba de igualdad de varianzas de los niveles de glicemia en el grupo control.

\begin{tabular}{cccc}
\hline $\mathrm{F}$ & g.l.1 & g.l.2 & Significación \\
\hline 3,032 & 3 & 16 & 0,060 \\
\hline
\end{tabular}

La prueba de Levenne indica que los niveles de la glicemia en la muestra control con respecto al tiempo mostraron diferencias no significativas $(p>0,05)$ entre las varianzas muestrales, lo que indica que las varianzas entre los tratamientos son iguales. 
Tabla 9. Pruebas de los efectos entre tratamientos

\begin{tabular}{cccc}
\hline & & Etiqueta del valor & $\mathrm{N}$ \\
\hline Inicio & 1 & inicio & 5 \\
& 2 & mes 1 & 5 \\
& 3 & mes 2 & 5 \\
& 4 & mes 3 & 5 \\
\hline
\end{tabular}

Tabla 10. Reducción de la hiperglicemia en grupo control

\begin{tabular}{cc}
\hline Fuente & Significación \\
\hline Modelo corregido & 0,110 \\
Intersección & 0,000 \\
inicio & 0,110 \\
Total corregida & \\
\hline
\end{tabular}

La prueba de efectos entre tratamientos en el grupo control indica que existe una interacción entre los tratamientos $(0,000<0,05)$, pero que las diferencias no son significativas en la reducción de la hiperglicemia tipo 2 cuando se comparan los niveles de glicemia inicial con los observados en cada uno de los 3 meses que duró el tratamiento con yogurt simbiótico y harina de maracuyá $(0,110>0,05)$.

Tabla 11. Medias de la glicemia marginales estimadas

\begin{tabular}{ccccc}
\hline & & \multicolumn{3}{c}{ Intervalo de confianza al 95 \% } \\
\cline { 3 - 5 } & & & Límite & Límite \\
Inicio & Media & Error típ. & inferior & superior \\
\hline inicio & 183,400 & 20,179 & 140,622 & 226,178 \\
mes 1 & 150,000 & 20,179 & 107,222 & 192,778 \\
mes 2 & 135,400 & 20,179 & 92,622 & 178,178 \\
mes 3 & 109,000 & 20,179 & 66,222 & 151,778 \\
\hline
\end{tabular}


Los intervalos de confianza muestras que existe interacción entre las variables analizadas son: tratamiento (con $500 \mathrm{ml} /$ día de yogurt simbiótico y harina de maracuyá, con dieta acostumbrada durante 3 meses) e Hiperglicemia (Reducción de los niveles de hiperglicemia tipo 2).

Tabla 12. Prueba de comparaciones múltiples de Duncan

\begin{tabular}{cccc}
\hline & & \multicolumn{2}{c}{ Subconjunto } \\
\cline { 3 - 4 } Inicio & $\mathrm{N}$ & 1 & 2 \\
\hline mes 3 & 5 & 109,00 & \\
mes 2 & 5 & 135,40 & 135,40 \\
inicio & 5 & 183,40 & 183,40 \\
mes 1 & 5 & & 150,00 \\
Significación & & 0,192 & 0,130 \\
\hline
\end{tabular}

La prueba de Duncan nos indica que a pesar de que la prueba inter sujetos nos indique que no hay diferencias, esta prueba nos muestra que sí existe, pero no significativamente, es decir, el mes 1 se diferencia con el de la prueba de control, mes 2 y mes 3 (grupo 1) y que el mes tres se diferencia de la prueba de control, mes 1 y mes 2 (grupo 2).

Tabla 13. Prueba de rangos de calificación sensorial

\begin{tabular}{cc}
\hline & Rango promedio \\
\hline Aroma & 2.33 \\
Color & 2.47 \\
Textura & 2.60 \\
Sabor & 2.60 \\
\hline
\end{tabular}

Tabla 14. Contrastación de hipótesis. Prueba de Friedman

\begin{tabular}{lc}
\hline $\mathrm{N}$ & 15 \\
\hline Chi-cuadrado & 4,714 \\
g.l. & 2 \\
Sig. asintótica & 0,194 \\
\hline
\end{tabular}

La prueba de significancia $(0,194>0,05)$ demuestra que no existen diferencias significativas en la aceptación del yogurt simbiótico con harina de maracuyá comparado con el yogurt natural, sin embargo, el yogurt simbiótico elaborado tiene propiedades hipoglicémicas por la harina de maracuyá. 


\section{DISCUSIÓN}

Según el análisis multivariante, el comportamiento de los niveles de la glicemia por efecto de la ingesta de 500 ml/día de yogurt simbiótico y harina de maracuyá con respecto al tiempo de tratamiento en el grupo de casos, muestran que existen diferencias significativas $(p<0,05)$ en los cuatro periodos donde se tomaron las medidas de la glicemia en personas con hiperglicemia tipo 2, evidenciándose una asociación entre estas dos variables.

Por otro lado, la prueba de efectos entre tratamientos en el grupo control indicó que si bien es cierto existe una interacción entre los tratamientos $(0,000<0,05)$, las diferencias son no significativas en la reducción de la hiperglicemia tipo 2, comparados con los niveles de reducción de la hiperglicemia, observados en cada uno de los tres meses que duró el tratamiento con yogurt simbiótico y harina de maracuyá $(0,110>0,05)$.

El efecto positivo guarda relación con lo reportado por Salgado, Bombarde, Mansi, Piedade y Meletti (2010) que realizaron ensayos con ratas Wistar con tres concentraciones (5, 10 y $15 \%$ ) de harina de cáscaras de maracuyá dentro de la dieta total. En ese estudio, encontraron una reducción de la tasa glucémica.

La dieta con un contenido del $5 \%$ fue la que presentó los mejores efectos en las ratas al reducir los niveles de glucosa en sangre en un $59 \%$ y un incremento en los niveles de glicógeno hepático del $71 \%$.

Esta situación demuestra el efecto de la cáscara de maracuyá en el metabolismo de los hidratos de carbono, lo cual influye positivamente en el control metabólico de la diabetes y ayuda en la prevención o el retraso de algunas complicaciones asociadas con esta enfermedad.

\section{CONCLUSIONES}

La harina de maracuyá es un alimento funcional que aporta principalmente elevada cantidad de fibra dietaria (fibra soluble, 3,50 g \% y 54,19 g \% de fibra insoluble), y antioxidantes naturales (18,358 $\pm 0,384 \mathrm{mgEAG} /$ gs de fenoles totales y 0,091 $\pm 0,012$ mgEC3G/ gs de antocianos totales), los cuales contribuyen a una alimentación saluda-ble complementarios a la dieta y propiedades hipoglucémicas.

El yogurt simbiótico con harina de maracuyá, según la prueba de significancia (0,194 > $0,05)$, demuestra que no existen diferencias significativas en la aceptación del yogurt simbiótico con harina de maracuyá comparado con el yogurt natural, ya que es del agrado de las personas adultas con hiperglicemia tipo 2.

El tratamiento con dosis de $500 \mathrm{ml}$ por día de yogurt simbiótico y harina de maracuyá, como apoyo nutricional a la dieta acostumbrada de personas con hiperglicemia tipo 2 (grupo de casos), produjo efectos positivos en la reducción entre el 8 \% y $9 \%$ de los valores cuantitativos de la hiperglicemia.

De este modo, existen diferencias significativas $(p<0,05)$ durante los tres meses de tratamiento, evidenciándose una asociación entre estas dos variables (consumo de yogur simbiótico y reducción de la hiperglice-mia tipo 2).

Existe interacción de la dieta y la reducción de la hiperglicemia tipo $2(0,000<0,05)$, sin embargo, con menor efectividad, cuando se comparan los niveles de glicemia inicial con 
los observados en cada uno de los tres meses que duró el tratamiento con yogurt simbiótico y harina de maracuyá $(0,110>0,05)$.

\section{REVISIÓN BIBLIOGRAFÍA}

Andersson, H. A. (2001). Health effects of probiotics and prebiotics: A literature review on human studies. Scand J Nutr 2001, 45, 5875.

Diplock, A.T. (1999). Scientific concepts of functional food in Europe. Consensus document. Br J Nutr, 81, 1-27.

Dongowski, G., Drzikova, B., Senge, B., Blochwitz, R., Gebhardt, E., y Habel, A. (2005). Comportamiento Reológico de las preparaciones B-glucano de productos de avena.

Espin, J. C., Soler, C., Wichers, H. J., y García, C. (2000). Anthocyanin-based natural colorants: A new source of antiradical activity for foodstuff. J. Agric. Food Chem, 48(1), 15881592.

Metchnikoff, E. (1907). Lactic acid as inhibiting intestinal putrefaction. In: The prolongation of life: Optimistic studies. London: W. Heinemman, 161-83.

Oldenburg, B., y Pijl, H. (2001). Abdominal obesity: metabolic complications and consequences for the liver. Ned Tijdschr Geneeskd, 145(27), 1290-4.

OMS. (2017). INFOBAE. Obtenido de https:// www.infobae.com/salud/2017/11/14/cadaseis-segundos-muere-en-el-mundo-unapersona-por-diabetes/

Salgado, J., Bombarde, T., Mansi, D., Piedade S., y Meletti. L. (2010). Effects of different concentrations of passion fruit peel (Passiflora edulis) on the glicemic control in diabetic rat. Ciencia y Tecnología de Alimentos, 30(3), 784-789. 